\title{
Development of transition region based methods for image segmentation
}

\author{
Priyadarsan Parida* \\ * Department of Electronics and Telecommunication Engineering, Veer Surendra Sai University of Technology, Burla, \\ Sambalpur,India \\ Advisor: Nilamani Bhoi
}

Date and location of PhD thesis defence: 9 February 2019, Veer Surendra Sai University of Technology.

Permanent link of thesis: http://hdl.handle.net/10603/240490

Received 8 August 2000; revised 8 January 2020; accepted 10 January 2020

\begin{abstract}
In this thesis, some transition region based segmentation approaches have developed to perform image segmentation for grayscale and colour images.

In computer vision and image understanding applications, image segmentation is an important pre-processing step. The main goal of the segmentation process is the separation of foreground region from background region. The segmentation approaches are application specific and do not work well for both grayscale and colour image segmentation. For any image consisting of foreground and background, some transition regions exist between the foreground and background regions. Effective extraction of transition region leads to a better segmentation result. Therefore, the doctoral thesis intends to efficient and effective transition region extraction approaches for image segmentation for both grayscale and colour images.
\end{abstract}

Key Words: Computer Vision, Image segmentation, Transition region, Local variance.

\section{Introduction}

In image processing and computer vision, image segmentation is an important step for different tasks such as detection, recognition, analysis etc. Since inception, a number of image segmentation approaches has been developed by various researchers which can be categorized as traditional approach such as thresholding, region based, boundary based etc. and hybrid approaches which is a combination of two or more traditional approach. Transition region-based thresholding is a kind of hybrid region based and thresholding based method which is used in recent days due to the following reasons:

- The transition region always exist for step-edge as well as non-step edge.

- Transition regions are located between object and background and around objects.

- Gray levels of the pixels in the transition region usually change frequently and intensively.

Correspondence to: priyadarsan.vssut@gmail.com

Recommended for acceptance by Dena Bazazian \& Xim Cerdá

ELCVIA ISSN: $1577-5097$

Published by Computer Vision Center / Universitat Autonoma de Barcelona, Barcelona, Spain

DOI: https://doi.org/10.5565/rev/elcvia.1176 
The transition regions for images were initially demonstrated by Gerbrands [1]. Using this transition region concept, many techniques such as LE [2], MLE [3], LGLD [4], RIB [5], etc. are developed for effective segmentation.

\section{Motivation and Objective}

The transition region based approaches developed so far are popular due to their simplicity and effective computation. When the transition region methods are applied to images containing texture background or overlapping intensities between objects and backgrounds, the pixels belonging to the background are classified as transition region. This result in ineffective transition region extraction leading to poor segmentation result. To overcome this, we have proposed transition region based methods which provide better transition regions. These proposed methods:

- Extract transition region for both single and multiple objects from images.

- Have better transition region extraction when there exist overlapping intensities between background and foreground.

- Extract transition region in presence of textured background.

- Provide continuity in the transition region.

It is found that, when the transition regions are extracted effectively from an image, the segmentation results are improved both quantitavely and qualitatively. Based on the aforementioned objectives, in the thesis we have proposed 8 different methods for effective transition region extraction followed by image segmentation for different types of photographic images for both gray and color images.

\section{Contributions}

The doctoral research work is focused on the development of transition region approaches both in spatial and wavelet domain for image segmentation. The algorithms developed are categorized as

(A) Grayscale transition region based segmentation approaches

(B) Colour image transition region based segmentation approaches

The details are summarized below.

Grayscale transition region based segmentation approaches: Five transition region based approaches are developed for grayscale image segmentation (i) Proposed method 1, (ii) Proposed method 2, (iii) Proposed method 3, (iv) Proposed method 4 and (iv) Proposed method 5.

(i) Proposed method 1: The Proposed method 1 [6] extracts transition region using local variance and global thresholding considered from the variance features. The method utilizes edge linking and morphological operations for object extraction. The method is intended for segmentation of single and multiple objects from the image. The method does not perform well when the object and background gray level intensities are overlapping in nature.

(ii) Proposed method 2: The Proposed method 2 [7] utilizes 2-dimensional Gabor filter and global thresholding considered from the Gabor features for transition region extraction. Further, it uses edge linking and morphological operations for object extraction. The method is developed for eradicating the drawbacks of Proposed method 1. The performance of this method degrades in the presence of background texture.

(iii) Proposed method 3: The Proposed method 3 [8] is a hybridization of Proposed method 1 and Proposed method 2 in the transform domain. For suppressing the background textures, the wavelet transform is 
utilized in Proposed method 3. Utilizing the transitional features extracted by applying Proposed method 1 and Proposed method 2, a wavelet feature image is generated. The transition region is extracted by applying Otsu thresholding on these feature image. Further, edge linking and morphological operations are applied on it to extract the objects.

(iv) Proposed method 4: The Proposed method 4 [9] also utilizes the wavelet transform along with uses local standard deviation and Otsu thresholding for transition region extraction. The method suppresses the texture and extracts an effectively continuous transition region achieving better segmentation result. The method does not utilize the edge linking operation as used in Proposed method 1, Proposed method 2 and Proposed method 3. It uses less number of morphological operations as compared to that of Proposed method 1 and Proposed method 2.

(v) Proposed method 5: The Proposed method 5 [10] uses fuzzy c-means clustering of local variance features along with Otsu thresholding for transition region extraction.

Colour image transition region based segmentation approaches: Here three transition region based colour image segmentation which is the extension of gray level transition region approaches are developed. The segmentation methods developed are (i) Proposed method 6, (ii) Proposed method 7 and (iii) Proposed method 8.

(i) Proposed method 6: The Proposed method 6 [11] is an extension of Proposed method 1 developed in RGB colour space.

(ii) Proposed method 7: The Proposed method 7 [9] is an extension of Proposed method 4 processed in CIELab colour space. The method is applied to one agricultural application i.e. worm separation from infected plant leaves.

(iii) Proposed method 8: The Proposed method 8 [12] is a hybrid two-stage segmentation process which uses the algorithm of Proposed method 1 and Proposed method 2. This method processes the colour image in two different colour models (RGB model and CIE-Lab) for object extraction. The Proposed method 8 is applied for underwater fish image segmentation.

\section{Brief Results}

The performance of the segmentation is qualitatively measured visually by looking at the ground truths as well as the segmentation masks generated from different segmentation approaches or by comparing the original image and the segmented result. The quantitative performance of segmentation results is compared via five performance measures as misclassification error (ME), false positive rate (FPR), false negative rate (FNR), Jaccard index (JI) and segmentation accuracy (SA). The developed methods for gray image segmentation are compared with different existing segmentation methods such as Otsu, Kapur, MET, LE [2], MLE [3], LGLD [4], RIB [5], NC [13] and LSICM [14]. Similarly, the developed methods for colour image segmentation are compared with colour image segmentation methods like CV [15], ACWE [16] CISGA[17]. The experimental results are demonstrated both quantitatively and qualitatively in the thesis for different types of images.

\section{Conclusion}

From the overall comparative analysis of the proposed methods along with existing approaches for both gray and colour images are examined and the results are presented in the thesis it can be concluded that:

- The Proposed method 1 works well for images with simple background.

- The Proposed method 2 extracts objects in case of overlapping intensities between background and foreground. 
- The Proposed method 3, Proposed method 4 and Proposed method 5 suppresses the background texture very well and retains the objects.

- The Proposed method 6 works well for images with simple background.

- The Proposed method 7 and Proposed method 8 works effectively for all types of images.

To conclude overall out of the 8 developed methods, the Proposed method 7 is the variation of Proposed method 4 applied to CIE-Lab colour space. So, for a given image (gray/colour) Proposed method 4 can be used to achieve better segmentation.

Moreover, the Proposed method 7 and the Proposed method 8 are applied for two application based methods such as worm separation from leaves and fish segmentation from underwater images.

\section{References}

[1] Y. J. J. Zhang, J. J. J. Gerbrands, "Transition region determination based thresholding," Pattern Recognition Letters, 12(1): 13-23, 1991.

[2] C. Yan, N. Sang, T. Zhang, "Local entropy-based transition region extraction and thresholding," Pattern Recognition Letters, 24(16): 2935-2941, 2003.

[3] Z. Li et al., "Modified local entropy-based transition region extraction and thresholding," Applied Soft Computing Journal, 11(8): 5630-5638, 2011.

[4] Z. Li, C. Liu, "Gray level difference-based transition region extraction and thresholding," Computers and Electrical Engineering, 35(5): 696-704, 2009.

[5] Z. Li et al., "Robust single-object image segmentation based on salient transition region," Pattern Recognition 52: 317-331, 2016.

[6] P. Parida, N. Bhoi, "Transition region based single and multiple object segmentation of gray scale images," Engineering Science and Technology, an International Journal, 19(3): 1206-1215, 2016.

[7] P. Parida, N. Bhoi, "2-D Gabor filter based transition region extraction and morphological operation for image segmentation," Computers \& Electrical Engineering, 62: 119-134, 2017.

[8] P. Parida, N. Bhoi, "Wavelet based transition region extraction for image segmentation," Future Computing and Informatics Journal, 2(2): 65-78, 2017.

[9] P. Parida, N. Bhoi, "Feature based transition region extraction for image segmentation: Application to worm separation from leaves," Future Computing and Informatics Journal, 3(2): 262-274, 2018.

[10] P. Parida, N. Bhoi, "Fuzzy clustering based transition region extraction for image segmentation," Engineering Science and Technology, an International Journal, 21(4): 547-563, 2018.

[11] P. Parida, N. Bhoi, P. Dewangan, "Colour image segmentation based on transition region and morphological operation," 2017 International Conference on Wireless Communications, Signal Processing and Networking (WiSPNET) 62: 1293-1297, 2017.

[12] P. Parida, N. Bhoi, "Dual transition region extraction based colour image segmentation: Application to fish image segmentation," Global Journal of Computer Science and Technology: F Graphics \& vision, 17(3): 21-29, 2017.

[13] J. Shi, J. Malik, "Normalized cuts and image segmentation," IEEE Transactions on Pattern Analysis and Machine Intelligence, 22(8): 888-905, 2000.

[14] K. Zhang et al., "A Level Set Approach to Image Segmentation With Intensity Inhomogeneity," IEEE Transactions on Cybernetics, 46(2): 546-557, 2016.

[15] P. Getreuer, "Chan-Vese Segmentation," Image Processing On Line 2: 214-224, 2012.

[16] T. F. Chan, L. A. Vese, "Active contours without edges," IEEE Transactions on Image Processing, 10(2): 266-277, 2001.

[17] Alessia Amelio, Clara Pizzuti, "A genetic algorithm for color image segmentation," in Lecture Notes in Computer Science (including subseries Lecture Notes in Artificial Intelligence and Lecture Notes in Bioinformatics), 7835: 314-323, 2013. 